\title{
Article \\ Function and Biomarkers of the Blood-Brain Barrier in a Neonatal Germinal Matrix Haemorrhage Model
}

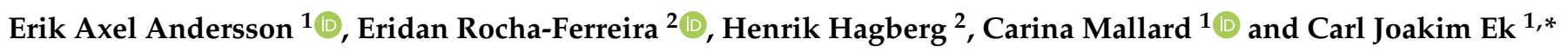 \\ 1 Institute of Neuroscience and Physiology, Sahlgrenska Academy, University of Gothenburg, \\ Medicinaregatan 11, 41390 Gothenburg, Sweden; axel.andersson@gu.se (E.A.A.); \\ carina.mallard@neuro.gu.se (C.M.) \\ 2 Institute of Clinical Sciences, Sahlgrenska Academy, University of Gothenburg, 41390 Gothenburg, Sweden; \\ eridan.rocha.ferreira@gu.se (E.R.-F.); henrik.hagberg@obgyn.gu.se (H.H.) \\ * Correspondence: joakim.ek@neuro.gu.se
}

Citation: Andersson, E.A.; RochaFerreira, E.; Hagberg, H.; Mallard, C.; Ek, C.J. Function and Biomarkers of the Blood-Brain Barrier in a Neonatal Germinal Matrix Haemorrhage Model. Cells 2021, 10, 1677. https:// doi.org/10.3390/cells10071677

Academic Editor: Andreina Schoeberlein

Received: 3 June 2021

Accepted: 29 June 2021

Published: 2 July 2021

Publisher's Note: MDPI stays neutral with regard to jurisdictional claims in published maps and institutional affiliations.

Copyright: (c) 2021 by the authors. Licensee MDPI, Basel, Switzerland. This article is an open access article distributed under the terms and conditions of the Creative Commons Attribution (CC BY) license (https:// creativecommons.org/licenses/by/ $4.0 /)$.

\begin{abstract}
Germinal matrix haemorrhage (GMH), caused by rupturing blood vessels in the germinal matrix, is a prevalent driver of preterm brain injuries and death. Our group recently developed a model simulating GMH using intrastriatal injections of collagenase in 5-day-old rats, which corresponds to the brain development of human preterm infants. This study aimed to define changes to the blood-brain barrier (BBB) and to evaluate BBB proteins as biomarkers in this GMH model. Regional BBB functions were investigated using blood to brain ${ }^{14} \mathrm{C}$-sucrose uptake as well as using biotinylated BBB tracers. Blood plasma and cerebrospinal fluids were collected at various times after GMH and analysed with ELISA for OCLN and CLDN5. The immunoreactivity of BBB proteins was assessed in brain sections. Tracer experiments showed that GMH produced a defined region surrounding the hematoma where many vessels lost their integrity. This region expanded for at least $6 \mathrm{~h}$ following GMH, thereafter resolution of both hematoma and re-establishment of BBB function occurred. The sucrose experiment indicated that regions somewhat more distant to the hematoma also exhibited BBB dysfunction; however, BBB function was normalised within 5 days of GMH. This shows that GMH leads to a temporal dysfunction in the BBB that may be important in pathological processes as well as in connection to therapeutic interventions. We detected an increase of tight-junction proteins in both CSF and plasma after GMH making them potential biomarkers for GMH.
\end{abstract}

Keywords: germinal matrix haemorrhage; blood-brain barrier; neonatal brain; tight-junctions; preterm

\section{Introduction}

Improved obstetric and neonatal care has contributed to increasing survival rates of extremely preterm infants (defined as infants born before pregnancy week 28). However, these infants have a high risk of suffering from germinal matrix haemorrhage (GMH). The incidence of GMH is around $20 \%$ in low-birth-weight $(<1500 \mathrm{~g})$ preterms, and as high as $35-40 \%$ in very low birth-weight preterms $(<750 \mathrm{~g})$, and, thus, is a major cause of morbidity and death in these groups of infants [1]. GMH involves the rupture of blood vessels in the germinal matrix, a specialised brain area during development rich in neurons and glia. GMH occurs mostly in the first few days after birth in preterm infants, with $50 \%$ within $6 \mathrm{~h}$ of birth [2]. The pathophysiology of GMH is still somewhat unclear but is thought to be multifactorial. One hypothesis often put forward is that germinal matrix vessels are more fragile than other vessels of the brain and thus more prone to rupture. There is evidence that completion of the neurovascular unit occurs later in this region, with less coverage of both astrocytic end-feet and pericytes [3,4], suggesting the particular vulnerability of this region. Additionally, regulation of cerebral blood flow (CBF) that is normally tightly controlled by auto-regulatory mechanisms is not fully developed in preterm infants [5]. Thus, shear flow stress on blood vessels from fluctuations of CBF 
combined with lesser supportive structures of blood vessels in the GM region may be part of the pathogenesis. This is supported by the fact that stabilising the CBF in infants can reduce the risk of GMH [6]. Other risk factors such as hypoxia, hypoglycaemia, and inflammation are yet to be clarified. If the haemorrhage breaks the neuroependyma and then extends into the brain ventricle, it is termed GMH-IVH, which is associated with a worse neurodevelopmental outcome and higher mortality [7]. This, in turn, is often linked to difficulties in cerebrospinal fluid drainage with shunting of cerebrospinal fluid (CSF), which poses its own risks, necessary in order to manage these complications [8]. One specialisation of the cerebrovasculature is its ability to tightly control the inward and outward passage of molecules between blood and brain. This concept is generally known as the blood-brain barrier (BBB), although the term is slightly misnamed since it encompasses not only barrier mechanisms but also specialised transport systems that will enhance entry of some blood solutes such as nutrients into the brain. Still, the blood-brain barrier is best recognised for keeping blood solutes out of the brain, making it difficult to develop effective pharmacotherapies for neurological disorders. There has been a general misconception that the BBB does not exist in the foetus. We and others have shown that indeed the BBB appears to form and become functional as soon as blood vessels penetrate into the brain [9-12], and the incompletion of the endothelial barrier is not thought to be underlying the pathophysiology of GMH [13]. Although measurements of BBB function are difficult to perform in human foetuses, the molecular make-up of brain blood vessels suggests a mature BBB phenotype in human foetuses [14]. Given that the vasculature is central in the pathophysiology and that BBB function is an important consideration in relation to potential therapies, we sought to determine vascular barrier function in relation to GMH. For this, we used a model of GMH in new-born rats, which we recently characterised, that shows several of the hallmarks of GMH in humans [15]. This model is based on postnatal day 5 rats, which are developmentally similar to preterm human infants born between approximately 26 to 32 weeks of gestation based on neurodevelopmental hallmarks, such as cortical development $[16,17]$. We used several approaches to determine BBB function following GMH and could see leaking vessels for days after GMH in the vicinity of the hematoma, as well as smaller changes in BBB function in more distant brain areas. In addition, we measured the levels of BBB proteins claudin-5 (CLDN5) and occludin (OCLN) in CSF and plasma following GMH in order to test their potential as biomarkers and found an increase in both CSF and plasma of these proteins.

\section{Materials and Methods}

\subsection{Animals}

Postnatal day 5 (PND5) Wistar rat pups were bred in-house at the Laboratory for Experimental Biomedicine of Gothenburg University (parents were sourced from Janvier Labs, Le Genest-Saint-Isle, France) and maintained under normal housing conditions with a $12 \mathrm{~h}$ light/dark cycle and free access to water and standard laboratory fodder. Animals of both sexes and different litters were used for the experiments, and care was taken to minimise the number of animals used and to maintain an even sex balance in all experimental and control groups. All experiments were approved by the Gothenburg Committee of the Swedish Animal Welfare Agency (Application nos. 825-2017) and performed in accordance with the ARRIVE guidelines. A total of 91 animals were used throughout the study, and the number of animals used per experiment is specified in the Methods and Results.

\subsection{Germinal Matrix Haemorrhage (GMH)}

GMH was induced in PND5 rats as previously described; this experimental approach induces brain injury, particularly in striatum, and the surrounding white matter [15]. In summary, isoflurane-anaesthetized ( $5 \%$ induction, $1.5 \%$ maintenance) rat pups were slowly injected with collagenase VII (Sigma-Aldrich, Saint-Louis, MO, USA) at a dose of $0.3 \mathrm{U} / \mathrm{mL}$ into the medial striatum of the right hemisphere, in proximity to the germinal matrix. Two $\mu \mathrm{L}$ infusions were made with a $27 \mathrm{G}(0.4 \mathrm{~mm})$ needle connected to a Hamilton syringe fitted 
in an infusion pump (CMA/100 microinjection pump) set to a flow rate of $1 \mu \mathrm{L} / \mathrm{min}$. The procedure typically lasted for 2-5 min. After infusions, the animals were allowed to recover on a heating pad set to $35{ }^{\circ} \mathrm{C}$ and then returned to their dams. All animals survived the GMH-model and until the end of experimentation.

\subsection{Functional Blood-Brain Barrier Assessment, Visible Tracers}

To assess BBB function in pups after $\mathrm{GMH}$, we performed BBB tracer experiments to detect vascular leakage of tracers at the individual blood vessel level. We used lowmolecular-weight biotin-ethylene-diamine (neurobiotin, $286 \mathrm{Da}$, Vector Laboratories, CA, USA) and a larger biotin-dextran (BDA, 10,000 Da, ThermoFisher, MA, USA). Doses and tracers were decided from our previous studies $[10,18]$. At various times after GMH $(2 \mathrm{~h}$, $6 \mathrm{~h}, 24 \mathrm{~h}$ and 5 days), animals were injected with tracers dissolved in saline; neurobiotin was injected ip at a concentration of $2.0-2.5 \mathrm{mg}$ per animal, and the same dose of BDA was slowly injected retro-orbitally under full anaesthesia. Three animals were used per tracer and time-point. Animals were euthanised with an overdose of pentobarbital at either $20 \mathrm{~min}$ (neurobiotin) or at $10 \mathrm{~min}$ (BDA) after the injection. The heart was punctured, and the brain immediately dissected out. The brain was placed on a flat surface, and the posterior part of the hemispheres removed with a razor blade, so the hematoma was in the middle of the remaining part of the cortex. The brain was then immediately immersed in cold buffered fixative (Histofix; Histolab, Gothenburg, Sweden) and left for $24 \mathrm{~h}$ at $4{ }^{\circ} \mathrm{C}$. The brains were embedded in $4 \%$ agarose and $60 \mu \mathrm{m}$ coronal sections were cut in a Leica 1200 VT vibratome (Leica Biosystems, Wetzlar, Germany) and stored in PBS until analysis.

\subsection{Visualisation of Tracers}

The injected biotinylated tracers were visualised in $60 \mu \mathrm{m}$ brain sections using $3^{\prime}$ diaminobenzidine (DAB). In between the different steps below, sections were washed with PBS containing $0.25 \%$ Triton $\mathrm{X}-100$. In short, residual agarose was removed from the sections before endogenous peroxidase was blocked by incubation with $1 \% \mathrm{H}_{2} \mathrm{O}_{2}$ in PBS and incubated overnight with the Vectastain Elite ABC HRP kit (Vector Laboratories). Sections were then incubated with DAB dissolved in a sodium acetate mixture with $\beta$ D-glucose, ammonium chloride, nickel sulphate and $\beta$-glucose-oxidase before they were mounted in water-based CC/Mount (Sigma-Aldrich, MO, USA). DAB-stained sections were photographed with a BX60 microscope equipped with a TH4-200 light-source using the cellSens software (Olympus, Tokyo, Japan), larger tiled and stitched images of entire sections were acquired with a Zeiss AxioImager Z2 equipped with an MRc AcioCam using the ZEN Blue software (Zeiss, Oberkochen, Germany).

\subsection{Quantification of Leaking Blood Vessels and Hematoma Size}

The size of the hematoma and the number of leaking blood vessels outside the hematoma were quantified in tiled/stitched images of entire DAB-stained brain sections from animals injected with the neurobiotin- and BDA-tracers using the Fiji build of ImageJ [19]. Leaking blood vessels were identified based on the extravasated tracer residue surrounding them. Leaking blood vessels in the sections were marked with the cell counter plugin, and the size of the hematoma was measured by encircling a region of interest around the hematoma. Additionally, the distance from the edge of the hematoma to the furthest leaking vessel was measured in each brain, using two sections per animal (hippocampal and striatal level), and the results averaged. $n=3$ per time-point.

\subsection{Functional Blood-Brain Barrier Assessment by Sucrose Extravasation}

A more quantitative assessment of regional BBB function was performed by measuring sucrose blood to brain uptake at $2 \mathrm{~h}, 6 \mathrm{~h}, 24 \mathrm{~h}$ and 5 days after GMH, together with control animals aged 5, 6 and 10 days, as we have previously described [20]. Briefly, ${ }^{14} \mathrm{C}$-labelled sucrose was injected ip at a dose of $2 \mu \mathrm{CI}$ per animal and animals killed at $30 \mathrm{~min}$ after injection with an overdose of pentobarbital. Blood was collected from the heart and plasma 
separated by centrifugation. Different regions of the left and right brain hemispheres were dissected out, where we specifically separated areas outside the hematoma in the ipsilateral hemisphere as well as areas more distant to hematoma in both hemispheres. Peri-hematoma regions were collected at least one millimetre from the hematoma to ensure no ruptured vessels were included to test BBB function outside the region of cerebrovascular ruptures. One animal was excluded from the study as the bleeding had reached the left hemisphere. Brain tissues and plasma were collected in pre-weighed scintillation vials, dissolved with $0.5 \mathrm{~mL}$ Solvable (PerkinElmer, MA, USA) overnight at $37^{\circ} \mathrm{C}$ and after visual assertion that tissues were dissolved, $5 \mathrm{~mL}$ of Ultima Gold scintillation cocktail (PerkinElmer) was added to each vial. The radioactivity in each vial was determined by liquid scintillation counting using a Tri-Carb 4910TR (PerkinElmer) and calculated as dpm/g tissue and a ratio between brain samples and plasma calculated as a measure of BBB permeability with blood space corrections of brain samples as in a previous study [21]. $n=7$ (control groups) or 6 (GMH groups) for all time-points.

\subsection{Fluorescent Immunohistochemistry and 3D Imaging}

For fluorescent immunohistochemistry, agarose was removed, and the sections were permeabilised in PBS/0.25\% Triton X-100 for $3 \mathrm{~h}$ before blocking in $4 \%$ donkey serum for $1 \mathrm{~h}$ at RT, followed by incubation with primary antibody mouse-anti-rat CLDN5 (ThermoFisher, $4 \mathrm{C} 3 \mathrm{C} 2$, diluted $1 / 250$ in PBS) for two nights at $4{ }^{\circ} \mathrm{C}$. Followed by incubation with AlexaFluor donkey-anti-mouse 488 (Invitrogen, MA, USA, diluted 1/250) and streptavidin-conjugated Texas Red (Vector Laboratories) $\mathrm{O} / \mathrm{N}$ at $4{ }^{\circ} \mathrm{C}$. Sections were mounted in ProLong Gold Antifade (ThermoFisher). Z-stacks of blood vessels in fluorescent sections were acquired with a Zeiss LSM 800 confocal microscope and 3D-imaged in ZEN blue.

\subsection{Levels of Tight-Junction Proteins in CSF and Plasma after GMH}

For all time-points after GMH and time-matched control ( 2 h, 6 h, 24 h and 5 days), animals were euthanised with a lethal overdose of pentobarbital. Blood was collected by cardiac puncture with EDTA-treated syringes and centrifuged at $2000 \times \mathrm{g}$ for five min to separate the plasma. Cerebrospinal fluid was collected via glass capillaries from the cisterna magma and was checked for blood contamination [21]; no contamination was detected in any sample. Samples were placed on dry ice directly after collection and later long-term stored at $-80{ }^{\circ} \mathrm{C}$ before analysis. The levels of tight-junction proteins were measured in plasma and CSF from GMH and control animals using commercially available ELISA kits for OCLN (Cusabio, Wuhan, China) and CLDN5 (Nordic BioSite, Stockholm, Sweden) following the manufacturer's instructions. Diluted samples and a series of standards in duplicates were incubated on antibody-coated plates before stepwise incubation with a secondary antibody, horseradish peroxidase-avidin, a 3,3',5,5'-tetramethylbenzidine substrate and the stop-solution. The optical density was determined in a Spectramax Plus microplate reader (San Jose, CA, USA) set to $450 \mathrm{~nm}$ with $540 \mathrm{~nm}$ wavelengthcorrection (OCLN) or $450 \mathrm{~nm}$ (CLDN5) and the protein concentration was calculated from the standard curve. CSF was diluted 10 times and plasma 20 times, and samples from the same animals were analysed for both proteins, $n=6$ (control groups) or 7 (GMH-groups) for all time-points.

\subsection{Statistics and Graph}

Statistical analyses were made using GraphPad Prism version 9.00 for Windows (GraphPad Software, CA, USA). Analyses include one-way ANOVA with Dunnett's multiple comparisons test or the Holm-Š́dák multiple comparison tests as well as paired and unpaired $t$-tests. Levels of tight-junctional proteins between sexes were analysed by two group comparisons adjusting for time as a factor (Qlucore Software). Figures were designed in Affinity Photo and Designer (Serif Europe, West Bridgford, United Kingdom). Data are presented as mean \pm SD. 


\section{Results}

\subsection{Functional Blood-Brain Barrier Assessment Using Molecular Tracers}

Collagenase injection in the medial striatum gives rise to GMH-like bleeding around the injection site. The bleed was macroscopically visible before and after vibratomesectioning (Figure 1a). To measure characteristics of the hematoma, entire $60 \mu \mathrm{m}$ brainsection sections from animals injected with tracer-molecules BDA or neurobiotin at different time-points after GMH were DAB-visualised and imaged in larger tiled and stitched images. The number of tracer-leaking blood vessels was quantified and compared in both hemispheres. Figure $1 \mathrm{~b}$ shows representative images from both hemispheres at all time-points with leaking blood vessels marked with arrows. Both tracers gave similar staining, and both neurobiotin ( 2 and $6 \mathrm{~h}$ ) and BDA ( $24 \mathrm{~h}$ and five days) are shown. All tracer-stained blood residues were manually delineated, and the contralateral hemisphere compared to the ipsilateral hemisphere (Figure 1c). The average size of the hematoma in the ipsilateral hemisphere was $0.89 \pm 0.29 \mathrm{~mm}^{2}$ at $2 \mathrm{~h}$, with an increase to $2.38 \pm 0.26 \mathrm{~mm}^{2}$ at $6 \mathrm{~h}(p=0.010), 18 \mathrm{~h}$ later the hematoma was reduced to $1.07 \pm 0.61 \mathrm{~mm}^{2}(p=0.014)$. By five days after GMH, no hematoma was visible in any section ( $p=0.019$ compared to $24 \mathrm{~h}$ ). The number of leaking blood vessels was $35 \pm 11$ in the ipsilateral hemisphere $2 \mathrm{~h}$ after $\mathrm{GMH}$, and $47 \pm 8$ at $6 \mathrm{~h}$, which was reduced to $19 \pm 5$ by $24 \mathrm{~h}(p=0.0 .012)$, while no leaking vessel could be seen at five days post-GMH, $p=0.039$ compared to $24 \mathrm{~h}$ (Figure $1 \mathrm{~d}$ ). No hematoma or leaking vessels were observed either in control animals or in the contralateral hemispheres at $2 \mathrm{~h}, 6 \mathrm{~h}, 24 \mathrm{~h}$ or five days after GMH, except in one animal at $6 \mathrm{~h}$ after GMH where the bleed had infiltrated to the border of the contralateral hemisphere. Sections from animals that had undergone GMH but not injected with the tracer showed no labelling. The average distance from the edge of the hematoma to the most distant leaking vessel was $0.33 \pm 0.02 \mathrm{~mm}$ at $2 \mathrm{~h}$, which increased to $0.67 \pm 0.03 \mathrm{~mm}$ at $6 \mathrm{~h}(p \leq 0.001)$, followed by a decrease to $0.37 \pm 0.05 \mathrm{~mm}$ at $24 \mathrm{~h}(p \leq 0.001)$ (Figure $1 \mathrm{e}) . n=3$ per time-points.

\subsection{Regional Sucrose Permeability Across Blood-Brain Barrier after GMH}

The effects of GMH on the BBB was further quantified by measuring the permeability of ${ }^{14} \mathrm{C}$-sucrose. To achieve regional measurements, the brain was dissected into four parts (Figure 2a), and the anterior part of the ipsilateral hemisphere of GMH-animals was further divided into two parts termed peri-hematoma 1 and 2 (Figure 2b). Since blood flow is likely to be compromised in the hematoma, we did not perform analysis in this area and focused on regions around the hematoma where we did not detect leaking vessels from tracer studies. For control animals, the brain was divided into two posterior (ipsi/contra) and two anterior parts (ipsi/contra). A significant increase in BBB permeability was detected $2 \mathrm{~h}$ after GMH in both peri-hematoma area 1 (GMH $0.10 \pm 0.005$ vs. controls $0.081 \pm 0.011$; $p=0.012$ ) and peri-hematoma area 2 (GMH $0.098 \pm 0.006$ vs. controls $0.081 \pm 0.01 ; p=0.019$ ). When comparing the posterior part of the hemispheres, no differences were found between control and GMH-animals (Figure 2c). We also calculated sucrose permeability ratios within each animal between the ipsi- and contra-lateral sides of the brain. Higher BBB permeability ratios were found at $2 \mathrm{~h}(1.08 \pm 0.03, p=0.002), 6 \mathrm{~h}(1.11 \pm 0.07, p=0.025)$ and $24 \mathrm{~h}(1.11 \pm 0.04, p=0.001)$ after GMH in peri-hematoma area 1 and at $2 \mathrm{~h}(1.06 \pm 0.03$, $p=0.004), 6 \mathrm{~h}(1.13 \pm 0.08, p=0.018)$, and $24 \mathrm{~h}(1.07 \pm 0.03, p=0.001)$ in peri-hematoma area 2. These ratios did not differ at 5 days after GMH, neither in the posterior part of hemispheres at any time-point in GMH animals nor in naïve control animals (Figure 2d, $n=7$ (control groups) or 6 (GMH groups) for all time-points. 
a
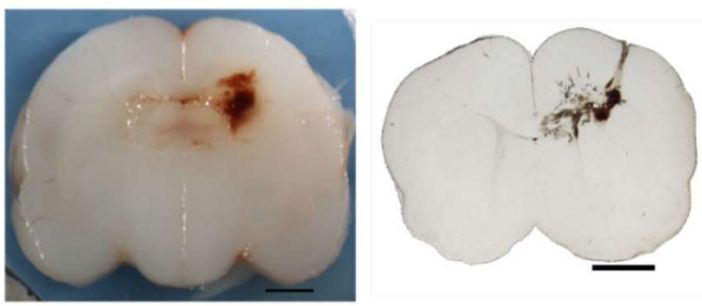

b
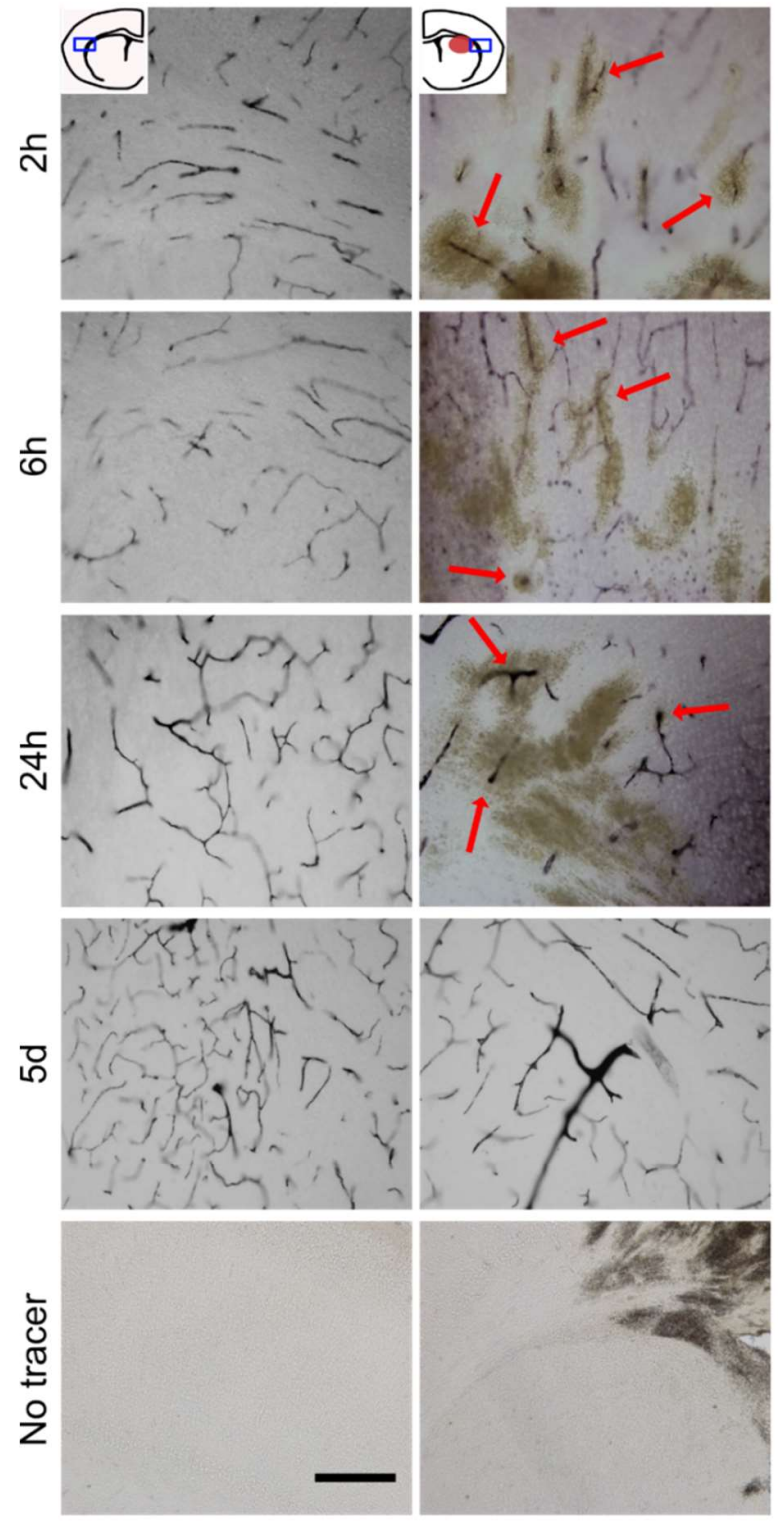

Contralateral

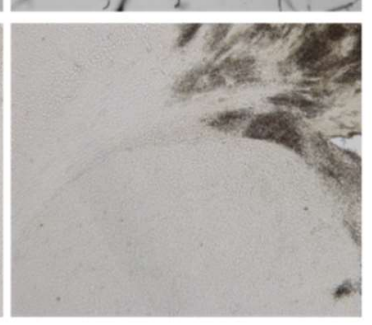

Ipsilateral
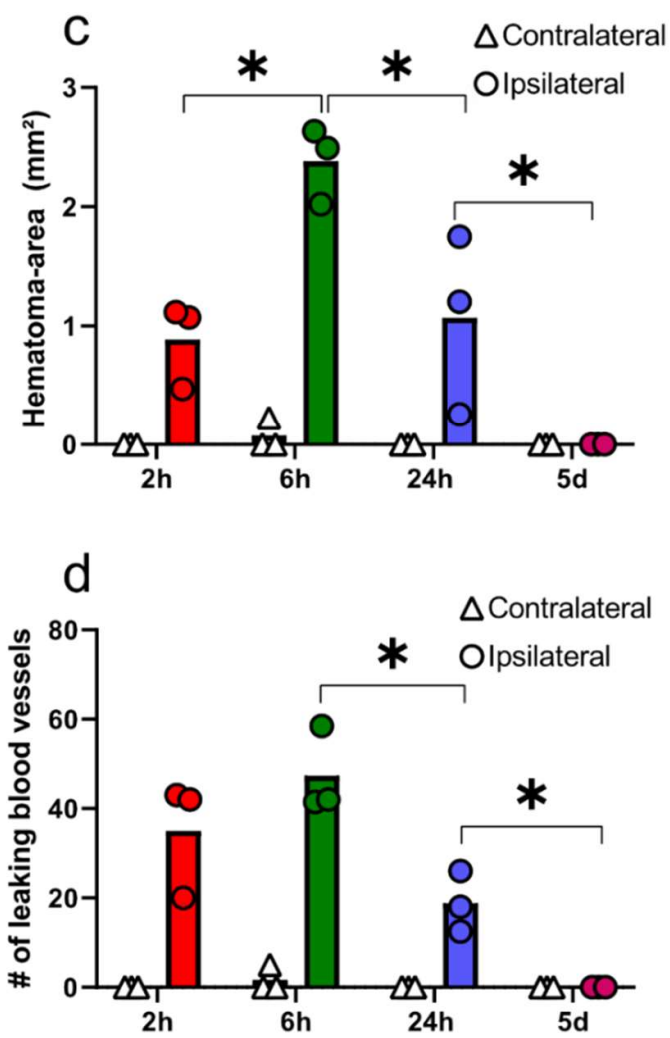

Figure 1. Functional blood-brain barrier assessment and quantification of leaking blood vessels using molecular tracers. (a) The hematoma is macroscopically visible in brains during processing and after sectioning. (b) Representative images of tracer distribution (red arrows) found in the area around the hematoma at different times following germinal matrix haemorrhage (GMH). Note that around the core hematoma, blood-brain barrier (BBB) function was compromised in some vessels up until $24 \mathrm{~h}$ following GMH. (c) The size of the hematoma after GMH. (d) Number of leaking blood vessels at different times after GMH in the peri-hematoma region. (e) Maximum distance to leaky blood vessels from the edge of the hematoma animals after GMH. One-way ANOVA with the Holm-Š́ídák multiple comparisons test, ${ }^{*} p \leq 0.05,{ }^{* * *} p \leq 0.001$. $n=3$ for all time-points, bar graphs represent mean values. Scale bars are $2 \mathrm{~mm}$ (a) and $100 \mu \mathrm{m}$ (b). 
a

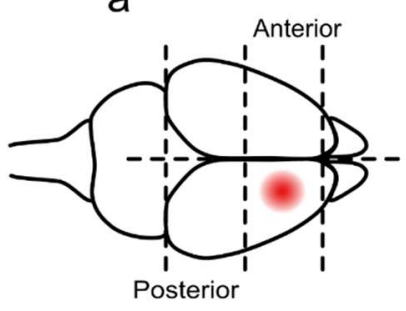

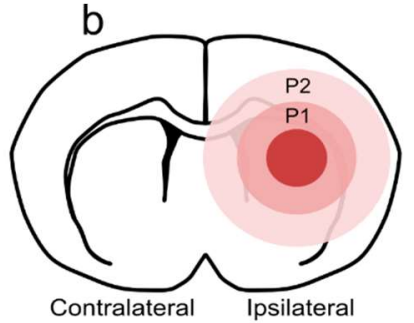

Contralateral Ipsilateral
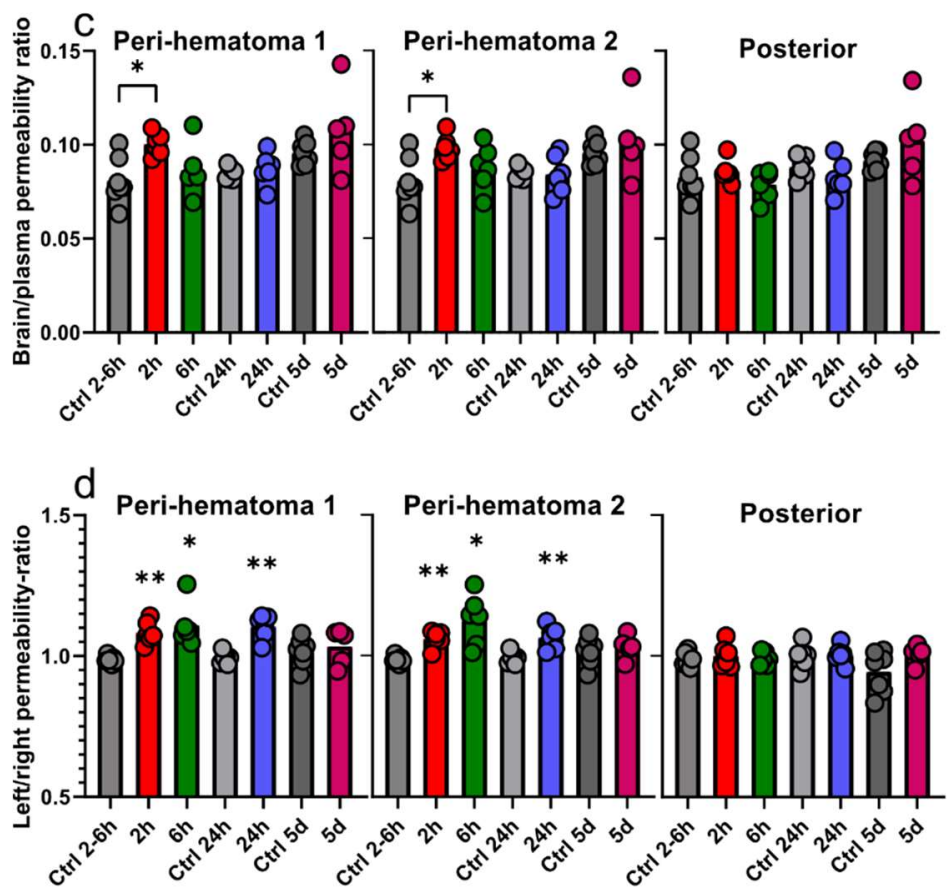

Figure 2. GMH induces spatial and temporal changes in BBB function. Regional BBB function was determined by sucrose permeability at different times after GMH. The regions of measurements are shown in (a) sagittal and (b) coronal (P1 = peri-hematoma 1, P2 = peri-hematoma 2). (c) The sucrose concentration ratios in the ipsilateral regions at $2 \mathrm{~h}, 6 \mathrm{~h}, 24 \mathrm{~h}$ and 5 days after GMH along with age-matched control animals. (d) The sucrose permeability concentration ratios between the ipsilateral and contralateral sides of the brain within animals. Unpaired $t$-tests and one-way ANOVA with Dunnett's multiple comparison test (c), paired $t$-tests (d), ${ }^{*} p \leq 0.05,{ }^{* *} p \leq 0.01 . n=7$ (control groups) or 6 (GMH groups) per time-points, bar graphs represent mean values.

\subsection{D Imaging of Leaking and Uninjured Brain Blood Vessels}

Blood vessels adjacent to the GMH-injury were further examined by 3D-imaging of entire vessels from z-stacks acquired by confocal microscopy of thick sections stained for CLDN5 and tracer. Images were taken in the peri-hematoma region and the corresponding area in the contralateral hemisphere. Vessels in the contralateral hemisphere $24 \mathrm{~h}$ after GMH showed coherent stretches of stained CLDN5 and tracer throughout the entire sections no injured vessels could be detected (Figure 3). In contrast, vessels that showed extravasation of tracer into adjacent brain parenchyma also showed discontinuous labelling of CLDN5. Five days after GMH, no leaking vessels could be detected in either hemisphere. 


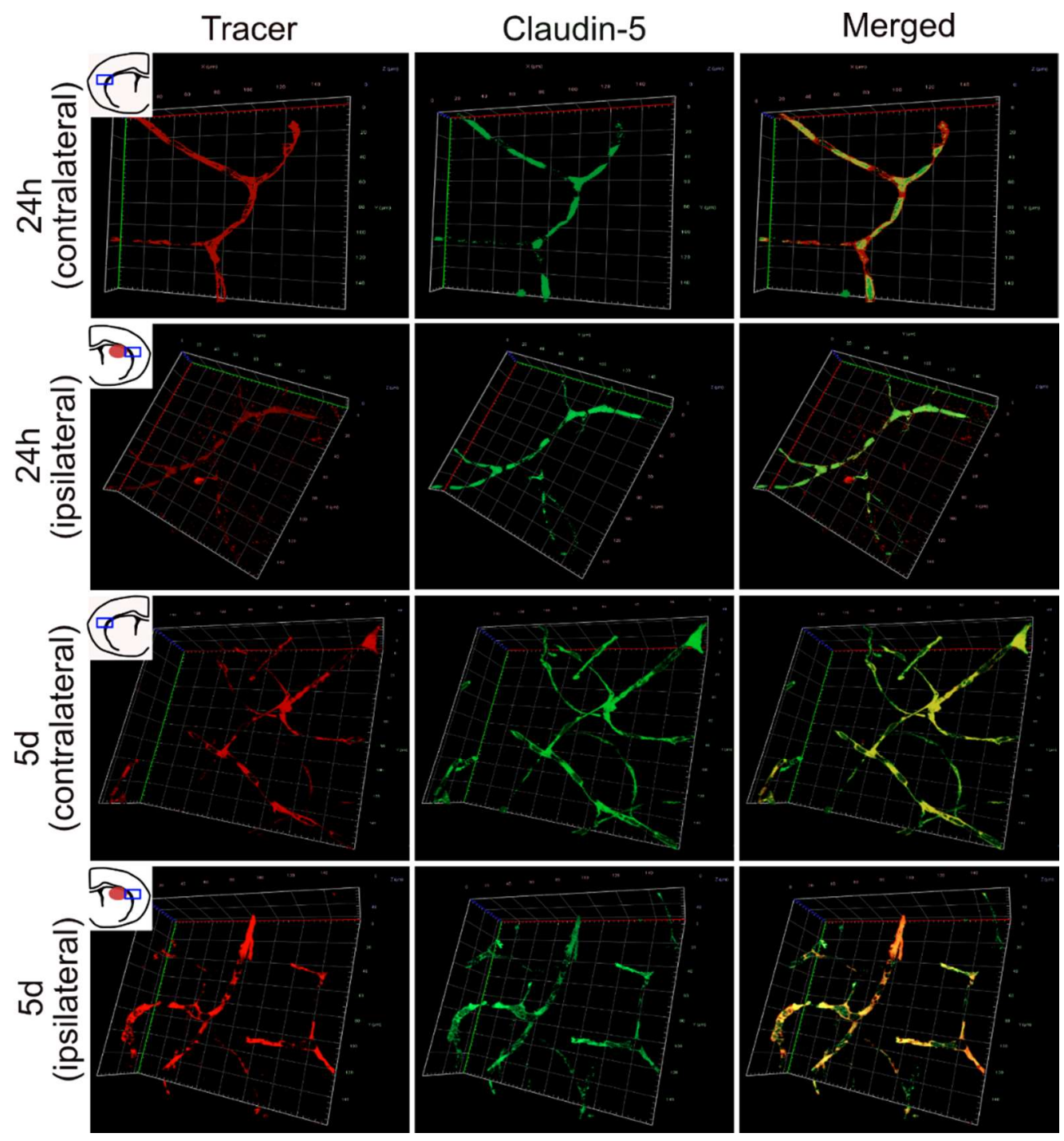

Figure 3. Three-dimensional imaging of tracer and CLDN5 in brains following GMH. Representative images of 3D-imaged z-stacks of vessels immunolabelled for claudin-5 (CLDN5) and tracer molecule taken in the peri-hematoma region of the ipsilateral hemisphere or the corresponding area in the contralateral hemisphere. At $24 \mathrm{~h}$ the CLDN5 labelling was discontinuous in vessels that also showed lost barrier integrity (tracer not contained within the vessel) in the ipsilateral hemisphere, while at five days, barrier integrity was re-established in blood vessels with continuous CLDN5 labelling and tracer contained within vessels. In the contralateral side of the brain, CLDN5 immunolabelling always remained continuous, and tracer remained within blood vessels. The $\mathrm{x}$ - and y-axes are $140 \mu \mathrm{m}$.

\subsection{BBB Proteins Are Increased in Plasma and CSF after GMH}

Tight-junction proteins were determined at $2 \mathrm{~h}, 6 \mathrm{~h}, 24 \mathrm{~h}$ and 5 days after GMH in blood plasma and CSF with ELISA-kits for CLDN5 and OCLN and compared to time-matched control groups (Figure 4a). CLDN5-levels in plasma were in the range of 10-20 ng/mL for most samples, with an increase $6 \mathrm{~h}$ after GMH $(20.1 \pm 4.4)$ compared to controls $(14.9 \pm 0.7$, $p=0.04)$. The protein was more abundant in CSF, with normal levels ranging between 45 and $80 \mathrm{ng} / \mathrm{mL}$, and was higher in GMH animals $(96.0 \pm 11.3)$ than controls $(67.4 \pm 12.3$ at $24 \mathrm{~h} ; p=0.009)$. OCLN-levels in plasma were lower in GMH animals (482.4 \pm 141.6$)$ compared to controls $(628.7 \pm 116.0)$ at $5 \mathrm{~d}(p=0.009)$. Several animals had elevated OCLN in CSF only $2 \mathrm{~h}$ after GMH, with significant increases at $6 \mathrm{~h}(547.6 \pm 121.4, p \leq 0.001)$ and $24 \mathrm{~h}$ post $\mathrm{GMH}(347.9 \pm 92.1, p=0.039)$ compared to controls (220.5 \pm 76.9$)$. CLDN5- and OCLN-levels were not different between GMH and control animals in either plasma or CSF, apart from higher levels in the five-day control group $(628.7 \pm 115.9, p=0.010)$ for OCLN 
in plasma. No sex differences were found in the levels of circulating OCLN or CLDN5 at any time-point in neither plasma nor CSF (Figure $4 b, p$-values ranged from $0.21-0.86$ ). See Supplementary Table S1 for average tight-junction proteins concentrations by sex in CSF and plasma. $n=6$ (control groups) or 7 (GMH-groups) for all time-points.

a
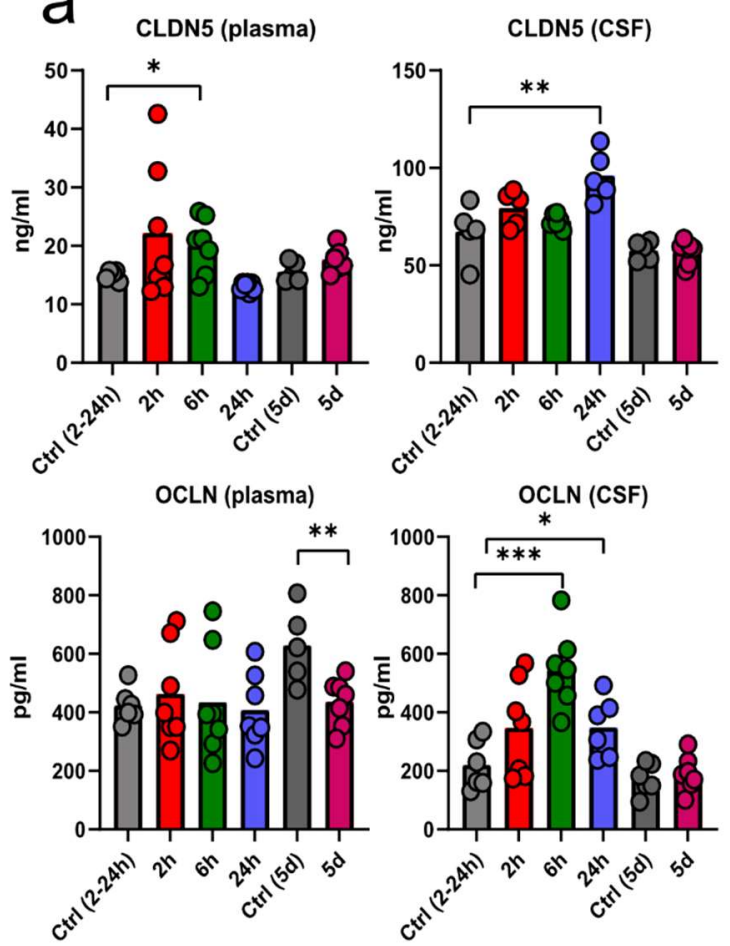

b
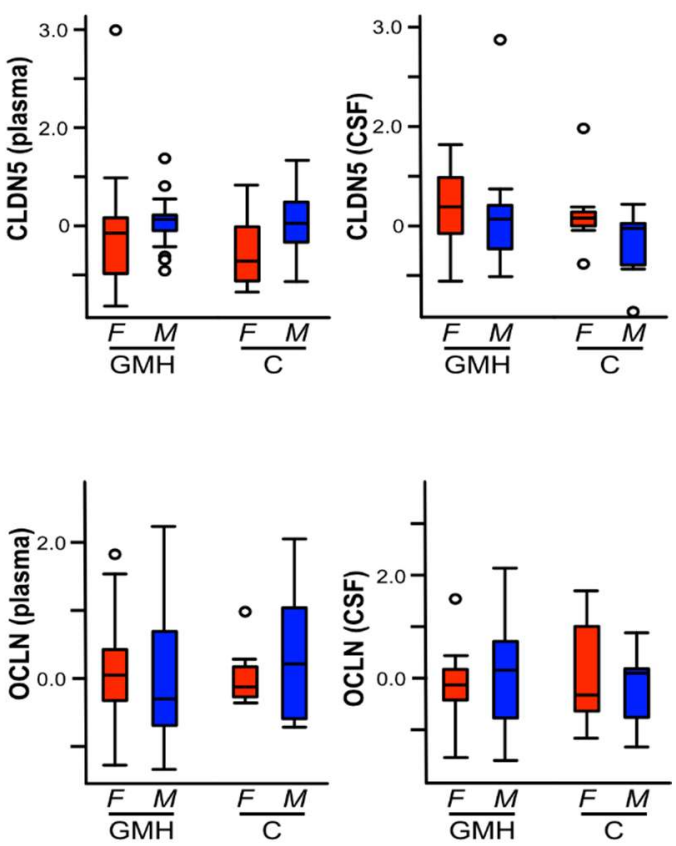

Figure 4. Time-dependent changes of tight junction protein levels in blood plasma and cerebrospinal fluid after GMH. The levels of CLDN5 and occludin (OCLN) was measured using ELISA at $2 \mathrm{~h}, 6 \mathrm{~h}, 24 \mathrm{~h}$ and five days after GMH, as well as time-matched control animals (a). Sex effect of CLDN5 and OCLN animals were investigated by pooling all samples from GMH and control (C) animals and adjusting levels for time. No difference was seen between male (M) and female (F) pups (b). Data was analysed by unpaired $t$-tests and one-way ANOVA with Dunnett's multiple comparison test, ${ }^{*} p \leq 0.05$, ${ }^{* *} p \leq 0.01$, and ${ }^{* * *} p \leq 0.001 . n=6$ (control groups) or 7 (GMH-groups) for all time-points. Bar graphs represent mean values; box-plots showing median, quartiles, range (whiskers) and outliers (open circles, $1.5^{*}$ quartile).

\section{Discussion}

GMH is a serious cause of death and disability, especially in low birth-weight premature infants, and there is a lack of treatments and diagnostic tools. Our group recently developed a rodent model for grade III and IV GMH using PND5 rats [15] to mimic this condition in the human preterm brain [16]. We had two objectives with this study. Firstly, we wanted to define regional changes to barrier function in the cerebrovasculature following GMH, as this is important both to understand the underlying pathophysiology and to aid potential therapeutic interventions that need to reach brain cells to be effective. Secondly, as BBB proteins have been shown to be potential biomarkers for brain vascular dysfunction in different settings of brain injury, we wanted to determine their levels in both CSF and plasma following GMH. We injected molecular tracers at different times after GMH and visualised these in the brain tissue together with the distribution of the key BBB transmembrane protein CLDN5 in brain blood vessels. The haemorrhage occurred rather quickly after collagenase injections and was visible in the pups within only 15-30 min following the injections. After $2 \mathrm{~h}$, a hematoma was visible in brain sections, and the vascular integrity within the hematoma appeared mostly devoid, although assessment of integrity at the individual blood vessel level is hampered by the hematoma and tracer distribution. In the area surrounding the hematoma, we found a zone up to around 300-350 $\mu \mathrm{m}$ 
where blood vessels show heterogeneous BBB function with some vessels that remain BBB competent while others have lost their barrier integrity with tracers leaking out quite freely. Outside this zone, all vessels retained tracers. There is a hematoma expansion phase up to at least $6 \mathrm{~h}$, accompanied by a greater number of vessels that lose BBB integrity even further from the hematoma. High-resolution imaging of these vessels in the peri-hematomal region showed that vessels leaking tracer also had lost CLDN5. At $24 \mathrm{~h}$ after GMH, the hematoma has shrunk by $45 \%$, and the number of leaky vessels is $40 \%$ less compared to $6 \mathrm{~h}$ after $\mathrm{GMH}$, consistent with an earlier observation that erythrocytes and iron are cleared by this time $[15,16]$. There was also a marked reduction of the area with leaky vessels in the peri-hematomal region between 6 and $24 \mathrm{~h}$. These data indicate vascular repair mechanisms starting to re-establish vascular integrity, resulting in a lesser number of leaky vessels and shrinkage of the hematoma by $24 \mathrm{~h}$. This repair phase continues with complete resolution of the hematoma by 5 days, and tracers are all visibly contained within the cerebral vasculature, clearly showing that vascular barrier function is restored. This is further supported by high-resolution 3D imaging of blood vessels showing continuous CLDN5 labelling and tracers retained within the vessels. The short duration between tracer injection and brain collection ensured that the observed tracer extravasation did really come from locally damaged vessels and had not diffused from other parts of the injury [18]. The small discrepancy between tracer studies and sucrose experiments on BBB function in the more distant peri-hematomal regions can most likely be explained by the higher sensitivity in the detection of sucrose in the parenchyma, which only pointed to a modest change in BBB function. The power of tracers is that we can assess BBB integrity at the blood vessel level that is not possible by measuring sucrose flux into the brain; thereby, these techniques complement each other. We previously showed that vascular integrity is lost in the hematomal region after collagenase injections, with many vessels showing fractured staining for both CLDN5 and laminin [15], but did not follow the temporal or spatial changes. Zhang and colleagues [22], who have used a similar GMH model to us in rats, albeit at P7, did measure increased extravasation of Evans blue after $\mathrm{GMH}$, as well as detecting lower levels of occludin, ZO- 1 and claudin- 3 at 3 days after GMH. Their measurements were performed in whole hemispheres, so it is difficult to judge where vascular integrity was lost or specifically where the loss of these proteins occurred. It should also be mentioned that it has later been established that claudin-3 does not confer BBB properties to cerebral blood vessels, where claudin- 5 is dominant, but its main sealing function is in epithelia [23]. They did see neuroprotection by both insulin-like growth factor 1 and fingolimod in this model with a reduction in BBB dysfunction showing that therapies directed towards the stabilisation of the cerebrovasculature could be beneficial in GMH $[22,24]$ and supporting the idea that secondary changes to vascular integrity are a driver of pathogenesis.

The haemorrhage results in the deposition of blood products, as well as giving rise to metabolites that could be toxic and drivers of secondary injury mechanisms. Some of these possible toxic compounds are thrombin, heme, iron and complement factors and pro-inflammatory compounds. For instance, Gao and colleagues used an IVH model in rats, where blood was directly injected into the lateral ventricles, showing that simultaneous thrombin injections resulted in hydrocephalus and increased levels of albumin in peri-ventricular areas indicative of loss of BBB function [25]. Ley and colleagues have made a series of studies using glycerol-induced IVH in rabbits that indicate that extracellular haemoglobin and its ferric state, methaemoglobin, are damaging and pro-inflammatory, and thus a central player in the secondary injury mechanisms [26-28]. The development of secondary injury is also driven by neuroinflammation as GMH leads to increases in inflammatory cytokines, microgliosis [29,30] and astrogliosis [15]. Since several of these secondary injury mechanisms could potentially also be damaging to endothelial functions or BBB modulatory, we wanted to further test BBB function in regions more distal to the hematoma. To achieve this, we used radio-tagged sucrose, which can be measured highly accurately in tissues to accurately quantify the BBB permeability [31]. This showed that we 
could detect a small increase in BBB permeability between GMH and control animals at $2 \mathrm{~h}$ after the GMH in peri-hematomal regions, but at later times no change was detected. To increase the resolution of these measurements, we also calculated permeability ratios within each animal where the collagenase-injected hemisphere was compared to the contralateral hemisphere. These ratios are consistently higher in the peri-hematomal regions between 2 and $24 \mathrm{~h}$ compared to contralateral areas, indicating an increase in BBB permeability, albeit the magnitude of change is small. The peri-infarct areas where we saw increases in BBB-permeability is also the same areas where the white- and grey-matter tissue loss are most pronounced in this model [15]. The ratios, however, are probably somewhat of an underestimate since oedema is likely occurring predominantly in the haemorrhagic hemisphere, diluting extracellular markers. In addition, ratios within animals should be interpreted with some caution since it is possible that the contralateral hemisphere is somewhat affected in this model. However, overall, our data do not suggest that BBB function is affected in the contralateral hemisphere of GMH animals as no change was detected in this hemisphere from control animals and, furthermore, in GMH animals, the ratios between hemispheres are equal across the uninjured posterior part of the hemispheres. A study using an adult model of brain haemorrhage, where blood was injected into a similar region of the brain to our model, was also not able to detect changes in BBB function in the contralateral hemisphere [32]. Similar to our tracer studies, the sucrose experiments indicated a normalisation of BBB function at 5 days after the haemorrhage.

We and others have shown that elevated levels of tight-junction proteins can be detected in the circulation in rodent models for neonatal hypoxia/ischemia (HI) [20] and adult middle-cerebral artery occlusion [33], as well as in adult human patients with stroke [34] and intracranial haemorrhage [35], making these proteins promising biomarkers for brain vascular dysfunction in different settings of brain injury. Furthermore, the levels of these proteins correlated with the brain injury in neonatal HI [20], while in stroke patients, levels predicted haemorrhagic transformation [34]. We, therefore, wanted to measure these proteins following GMH, where the cerebral vasculature is a central component of the injury process. After GMH, changed levels were detected in both plasma and CSF with the greatest increase in CLDN5 at $6 \mathrm{~h}$ in plasma and $24 \mathrm{~h}$ in CSF compared to control animals, and elevated OCLN in CSF at $6 \mathrm{~h}$ and $24 \mathrm{~h}$, concurring with our previous results from neonatal HI. Our previous results showed that the levels of circulating OCLN in plasma and CLDN5 in CSF are differentially changed by sex, with higher levels in males, which may correspond to that in models for neonatal $\mathrm{HI}$ results in more severe injuries in male animals [36,37]. In GMH, we found no sex-dependent influence in tight junction-protein levels in CSF nor plasma. While male infants seem to have a higher risk for developing GMH [38] and get a worse neurological outcome than females [39,40], there are, to our knowledge, no studies that investigate sex-dependency on injury in animal models of GMH. Thus, we can conclude that these proteins potentially could be used as biomarkers in the setting of GMH; however, they need to be further tested in other models of GMH, as well as in clinical samples, in order to evaluate their usefulness in general.

In conclusion, this study shows that the PND5 model of GMH gives rise to a core of haemorrhagic vessels and to a quite defined surrounding region where some vessels lose their integrity, and larger molecules appear to freely move out of vessels. Both these regions expand in the first few hours after the induction of GMH, along with an increased number of leaking vessels. However, rather soon, there is re-establishment of barrier function in blood vessels, with a $50 \%$ reduction in the number of leaking vessels at $24 \mathrm{~h}$, and by five days, only vessels with competent BBB function are present. The vasculature is also affected around the haemorrhagic region with a moderate increase in BBB permeability, which seems to normalise at five days after GMH. We can also report that GMH gives rise to increased levels of tight-junction proteins in both CSF and plasma, making them potential biomarkers for GMH damage that should be further evaluated. 
Supplementary Materials: The following are available online at https:/ / www.mdpi.com/article/10 .3390 / cells10071677/s1, Table S1: Average tight-junction concentrations separated for group and sex. OCLN and CLDN5 was measured in the blood and plasma of all animals at $2 \mathrm{~h},(3 \mathrm{~m}, 4 \mathrm{f}) 6 \mathrm{~h}(4 \mathrm{~m}, 3 \mathrm{f})$, $24 \mathrm{~h}(3 \mathrm{~m}, 4 \mathrm{f}), 5 \mathrm{~d}(4 \mathrm{~m}, 3 \mathrm{f}), 2-24 \mathrm{~h}$ controls $(3 \mathrm{~m}, 4 \mathrm{f})$, and $5 \mathrm{~d}$ controls $(3 \mathrm{~m}, 4 \mathrm{f}) . \mathrm{m}=$ male, $\mathrm{f}=$ female.

Author Contributions: C.J.E., C.M. and H.H. conceived and designed the study, supervised all aspects of the study and obtained funding. E.R.-F., E.A.A. and C.J.E. performed animal experiments. E.A.A. designed and performed ex vivo experiments, image- and statistical-analyses. Results were interpreted by E.A.A., C.J.E., H.H. and C.M. E.A.A. wrote the initial draft of the paper with input, revisions and approval from all authors. Correspondence to be addressed to C.J.E. All authors have read and agreed to the published version of the manuscript.

Funding: The authors disclosed receipt of the following financial support for the research, authorship and/or publication of this article: Rune and Ulla Amlövs Foundation, Herbert and Karin Jacobsons Foundation (E.A.A.). The Hasselblad Foundation (2020-2021) and the Åke Wibergs Foundation (M19-0660) (E.R.-F.). Swedish Research Council (VR-2017-01409); Åhlen Foundation (C.M.); Public Health Service at the Sahlgrenska University Hospital (ALFGBG-722491); Swedish Brain Foundation; (FO2019-0270) (C.M.). The Swedish Medical Research Council (VR (2019-01320), the Swedish Governmental Grant to Researchers at University Hospitals (ALFGBG-718591), ERA-NET (Contract: 0755101), Brain Foundation (2015-0004), and EU (Contract: 874721 Horizon 2020) (H.H.). Cerebral Palsy Alliance Australia, Stroke-Riksförbundet, and Wilhelm and Martina Lundgren Foundations (C.J.E.).

Institutional Review Board Statement: All animal experiments were approved by the Gothenburg Committee of the Swedish Animal Welfare Agency (Application nos. 825-2017) and performed in accordance with the ARRIVE guidelines.

Informed Consent Statement: Not applicable.

Data Availability Statement: The data behind the conclusions of this study are available from the corresponding author upon reasonable request.

Conflicts of Interest: The authors declare that there is no conflict of interest with respect to the research, authorship, or publication of this article.

\section{References}

1. Özek, E.; Kersin, S.G. Intraventricular hemorrhage in preterm babies. Turk Pediatri Ars. 2020, 55, 215-221. [CrossRef] [PubMed]

2. Back, S.A.; Miller, S.P. 60-Brain Injury in the Preterm Infant. In Avery's Diseases of the Newborn, 10th ed.; Gleason, C.A., Juul, S.E., Eds.; Elsevier: Philadelphia, PA, USA, 2018; pp. 879-896.e876.

3. El-Khoury, N.; Braun, A.; Hu, F.; Pandey, M.; Nedergaard, M.; Lagamma, E.F.; Ballabh, P. Astrocyte End-Feet in Germinal Matrix, Cerebral Cortex, and White Matter in Developing Infants. Pediatr. Res. 2006, 59, 673-679. [CrossRef]

4. Braun, A.; Xu, H.; Hu, F.; Kocherlakota, P.; Siegel, D.; Chander, P.; Ungvari, Z.; Csiszar, A.; Nedergaard, M.; Ballabh, P. Paucity of Pericytes in Germinal Matrix Vasculature of Premature Infants. J. Neurosci. 2007, 27, 12012-12024. [CrossRef]

5. Gilmore, M.M.; Stone, B.S.; Shepard, J.A.; Czosnyka, M.; Easley, R.B.; Brady, K.M. Relationship between cerebrovascular dysautoregulation and arterial blood pressure in the premature infant. J. Perinatol. Off. J. Calif. Perinat. Assoc. 2011, 31, 722-729. [CrossRef]

6. Perlman, J.M.; Goodman, S.; Kreusser, K.L.; Volpe, J.J. Reduction in intraventricular hemorrhage by elimination of fluctuating cerebral blood-flow velocity in preterm infants with respiratory distress syndrome. N. Engl. J. Med. 1985, 312, 1353-1357. [CrossRef]

7. Atienza-Navarro, I.; Alves-Martinez, P.; Lubian-Lopez, S.; Garcia-Alloza, M. Germinal Matrix-Intraventricular Hemorrhage of the Preterm Newborn and Preclinical Models: Inflammatory Considerations. Int. J. Mol. Sci. 2020, 21, 8343. [CrossRef] [PubMed]

8. Tubbs, R.S.; Banks, J.T.; Soleau, S.; Smyth, M.D.; Wellons, J.C.; Blount, J.P.; Grabb, P.A.; Oakes, W.J. Complications of ventriculosubgaleal shunts in infants and children. Child's Nerv. Syst. 2005, 21, 48-51. [CrossRef]

9. $\quad$ Ek, C.J.; Habgood, M.D.; Dziegielewska, K.M.; Saunders, N.R. Structural characteristics and barrier properties of the choroid plexuses in developing brain of the opossum (Monodelphis Domestica). J. Comp. Neurol. 2003, 460, 451-464. [CrossRef] [PubMed]

10. Ek, C.J.; Dziegielewska, K.M.; Stolp, H.; Saunders, N.R. Functional effectiveness of the blood-brain barrier to small water-soluble molecules in developing and adult opossum (Monodelphis domestica). J. Comp. Neurol. 2006, 496, 13-26. [CrossRef]

11. Nitta, T.; Hata, M.; Gotoh, S.; Seo, Y.; Sasaki, H.; Hashimoto, N.; Furuse, M.; Tsukita, S. Size-selective loosening of the blood-brain barrier in claudin-5-deficient mice. J. Cell Biol. 2003, 161, 653-660. [CrossRef] [PubMed]

12. Daneman, R.; Zhou, L.; Kebede, A.A.; Barres, B.A. Pericytes are required for blood-brain barrier integrity during embryogenesis. Nature 2010, 468, 562-566. [CrossRef] [PubMed] 
13. Ballabh, P. Intraventricular hemorrhage in premature infants: Mechanism of disease. Pediatr. Res. 2010, 67, 1-8. [CrossRef] [PubMed]

14. Ballabh, P.; Hu, F.; Kumarasiri, M.; Braun, A.; Nedergaard, M. Development of tight junction molecules in blood vessels of germinal matrix, cerebral cortex, and white matter. Pediatr. Res. 2005, 58, 791-798. [CrossRef] [PubMed]

15. Jinnai, M.; Koning, G.; Singh-Mallah, G.; Jonsdotter, A.; Leverin, A.-L.; Svedin, P.; Nair, S.; Takeda, S.; Wang, X.; Mallard, C.; et al. A Model of Germinal Matrix Hemorrhage in Preterm Rat Pups. Front Cell Neurosci 2020, 14, 535320. [CrossRef] [PubMed]

16. Semple, B.D.; Blomgren, K.; Gimlin, K.; Ferriero, D.M.; Noble-Haeusslein, L.J. Brain development in rodents and humans: Identifying benchmarks of maturation and vulnerability to injury across species. Prog. Neurobiol. 2013, 106-107, 1-16. [CrossRef] [PubMed]

17. Mallard, C.; Vexler, Z.S. Modeling Ischemia in the Immature Brain: How Translational Are Animal Models? Stroke 2015, 46, 3006-3011. [CrossRef] [PubMed]

18. Habgood, M.D.; Bye, N.; Dziegielewska, K.M.; Ek, C.J.; Lane, M.A.; Potter, A.; Morganti-Kossmann, C.; Saunders, N.R. Changes in blood-brain barrier permeability to large and small molecules following traumatic brain injury in mice. Eur. J. Neurosci. 2007, 25, 231-238. [CrossRef] [PubMed]

19. Schindelin, J.; Arganda-Carreras, I.; Frise, E.; Kaynig, V.; Longair, M.; Pietzsch, T.; Preibisch, S.; Rueden, C.; Saalfeld, S.; Schmid, B.; et al. Fiji: An open-source platform for biological-image analysis. Nat. Methods 2012, 9, 676-682. [CrossRef]

20. Andersson, E.A.; Mallard, C.; Ek, C.J. Circulating tight-junction proteins are potential biomarkers for blood-brain barrier function in a model of neonatal hypoxic/ischemic brain injury. Fluids Barriers CNS 2021, 18, 7. [CrossRef]

21. Mottahedin, A.; Smith, P.L.; Hagberg, H.; Ek, C.J.; Mallard, C. TLR2-mediated leukocyte trafficking to the developing brain. J. Leukoc. Biol. 2017, 101, 297-305. [CrossRef]

22. Lekic, T.; Flores, J.; Klebe, D.; Doycheva, D.; Rolland, W.B.; Tang, J.; Zhang, J.H. Intranasal IGF-1 Reduced Rat Pup Germinal Matrix Hemorrhage. Acta Neurochir. Suppl. 2016, 121, 209-212. [CrossRef]

23. Castro Dias, M.; Coisne, C.; Lazarevic, I.; Baden, P.; Hata, M.; Iwamoto, N.; Francisco, D.M.F.; Vanlandewijck, M.; He, L.; Baier, F.A.; et al. Claudin-3-deficient C57BL/6J mice display intact brain barriers. Sci. Rep. 2019, 9, 203. [CrossRef] [PubMed]

24. Rolland, W.B.; Krafft, P.R.; Lekic, T.; Klebe, D.; LeGrand, J.; Weldon, A.J.; Xu, L.; Zhang, J.H. Fingolimod confers neuroprotection through activation of Rac1 after experimental germinal matrix hemorrhage in rat pups. J. Neurochem. 2017, 140, 776-786. [CrossRef]

25. Gao, F.; Liu, F.; Chen, Z.; Hua, Y.; Keep, R.F.; Xi, G. Hydrocephalus after Intraventricular Hemorrhage: The Role of Thrombin. J. Cereb. Blood Flow Metab. 2013, 34, 489-494. [CrossRef] [PubMed]

26. Gram, M.; Sveinsdottir, S.; Ruscher, K.; Hansson, S.R.; Cinthio, M.; Åkerström, B.; Ley, D. Hemoglobin induces inflammation after preterm intraventricular hemorrhage by methemoglobin formation. J. Neuroinflamm. 2013, 10, 867. [CrossRef]

27. Agyemang, A.A.; Sveinsdóttir, K.; Vallius, S.; Sveinsdóttir, S.; Bruschettini, M.; Romantsik, O.; Hellström, A.; Smith, L.E.H.; Ohlsson, L.; Holmqvist, B.; et al. Cerebellar Exposure to Cell-Free Hemoglobin Following Preterm Intraventricular Hemorrhage: Causal in Cerebellar Damage? Transl. Stroke Res. 2017, 8, 461-473. [CrossRef] [PubMed]

28. Ley, D.; Romantsik, O.; Vallius, S.; Sveinsdóttir, K.; Sveinsdóttir, S.; Agyemang, A.A.; Baumgarten, M.; Mörgelin, M.; Lutay, N.; Bruschettini, M.; et al. High Presence of Extracellular Hemoglobin in the Periventricular White Matter Following Preterm Intraventricular Hemorrhage. Front. Physiol. 2016, 7, 330. [CrossRef] [PubMed]

29. Tang, J.; Chen, Q.; Guo, J.; Yang, L.; Tao, Y.; Li, L.; Miao, H.; Feng, H.; Chen, Z.; Zhu, G. Minocycline Attenuates Neonatal Germinal-Matrix-Hemorrhage-Induced Neuroinflamm. and Brain Edema by Activating Cannabinoid Receptor 2. Mol. Neurobiol. 2016, 53, 1935-1948. [CrossRef] [PubMed]

30. Wang, T.; Zhang, J.; Li, P.; Ding, Y.; Tang, J.; Chen, G.; Zhang, J.H. NT-4 attenuates neuroinflammation via TrkB/PI3K/FoxO1 pathway after germinal matrix hemorrhage in neonatal rats. J. Neuroinflamm. 2020, 17, 158. [CrossRef]

31. Ek, C.J.; D’Angelo, B.; Baburamani, A.A.; Lehner, C.; Leverin, A.L.; Smith, P.L.; Nilsson, H.; Svedin, P.; Hagberg, H.; Mallard, C. Brain barrier properties and cerebral blood flow in neonatal mice exposed to cerebral hypoxia-ischemia. J. Cereb. Blood Flow Metab. Off. J. Int. Soc. Cereb. Blood Flow Metab. 2015, 35, 818-827. [CrossRef] [PubMed]

32. Yang, G.-Y.; Betz, A.L.; Chenevert, T.L.; Brunberg, J.A.; Hoff, J.T. Experimental intracerebral hemorrhage: Relationship between brain edema, blood flow, and blood-brain barrier permeability in rats. J. Neurosurg. 1994, 81, 93. [CrossRef] [PubMed]

33. Pan, R.; Yu, K.; Weatherwax, T.; Zheng, H.; Liu, W.; Liu, K.J. Blood Occludin Level as a Potential Biomarker for Early Blood Brain Barrier Damage Following Ischemic Stroke. Sci. Rep. 2017, 7, 40331. [CrossRef]

34. Kazmierski, R.; Michalak, S.; Wencel-Warot, A.; Nowinski, W.L. Serum tight-junction proteins predict hemorrhagic transformation in ischemic stroke patients. Neurology 2012, 79, 1677-1685. [CrossRef] [PubMed]

35. Jiao, X.; He, P.; Li, Y.; Fan, Z.; Si, M.; Xie, Q.; Chang, X.; Huang, D. The Role of Circulating Tight Junction Proteins in Evaluating Blood Brain Barrier Disruption following Intracranial Hemorrhage. Dis. Markers 2015, 2015, 860120. [CrossRef]

36. Hill, C.A.; Fitch, R.H. Sex differences in mechanisms and outcome of neonatal hypoxia-ischemia in rodent models: Implications for sex-specific neuroprotection in clinical neonatal practice. Neurol. Res. Int. 2012, 2012, 867531. [CrossRef] [PubMed]

37. Hill, C.A.; Threlkeld, S.W.; Fitch, R.H. Early testosterone modulated sex differences in behavioral outcome following neonatal hypoxia ischemia in rats. Int. J. Dev. Neurosci. Off. J. Int. Soc. Dev. Neurosci. 2011, 29, 381-388. [CrossRef] [PubMed]

38. Mohamed, M.A.; Aly, H. Male Gender Is Associated With Intraventricular Hemorrhage. Pediatrics 2010, 125, e333-e339. [CrossRef] 
39. Kent, A.L.; Wright, I.M.R.; Abdel-Latif, M.E. Mortality and Adverse Neurologic Outcomes Are Greater in Preterm Male Infants. Pediatrics 2012, 129, 124-131. [CrossRef]

40. Tioseco, J.A.; Aly, H.; Essers, J.; Patel, K.; El-Mohandes, A.A.E. Male sex and intraventricular hemorrhage. Pediatr. Crit. Care Med. 2006, 7, 40-44. [CrossRef] 